\title{
Improved Power Conversion Efficiency of InGaN Photovoltaic Devices Grown on Patterned Sapphire Substrates
}

\author{
C. C. Yang, J. K. Sheu, C. H. Kuo, M. S. Huang, S. J. Tu, F. W. Huang, \\ M. L. Lee, Yu-Hsiang Yeh, X. W. Liang, and W. C. Lai
}

\begin{abstract}
The InGaN/sapphire-based photovoltaic (PV) cells with $\mathrm{Al}_{0.14} \mathrm{Ga}_{0.86} \mathrm{~N} / \mathrm{In}_{0.21} \mathrm{Ga}_{0.79} \mathrm{~N}$ superlattice structures that serve as absorption layers were grown on patterned sapphire substrates (PSSs). Under global air-mass 1.5 conditions, the shortcircuit current density, the open-circuit voltage, and the fill factor obtained from the PV cells were $1.21 \mathrm{~mA} / \mathrm{cm}^{2}, 2.18 \mathrm{~V}$, and 0.65 , respectively, corresponding to a conversion efficiency of $1.71 \%$. Compared with PV devices grown on flat sapphire substrates, the photocurrent of PSS-grown PV devices was enhanced by $26 \%$. The improved PV performance was attributable to the positive effects of the PSS on the material quality.
\end{abstract}

Index Terms-InGaN, patterned sapphire substrate (PSS), photovoltaic (PV).

\section{INTRODUCTION}

$\mathbf{S}$ INCE 2002, InGaN-based compounds for photovoltaic (PV) devices have been extensively discussed, mainly with regard to multiple quantum well [1], [2], p-i-n junction [3], [4], and short-period superlattice (SPS) designs [5], [6], due to their potential for applications in full-solar-spectrum absorption [7], [8]. The most common sapphire $\left(\mathrm{Al}_{2} \mathrm{O}_{3}\right)$ substrates have been widely applied to light-emitting and laser diodes [9] and show a great potential for mass production and affordable prices for future PV applications. Although InGaN semiconductors for light-emitting devices show a little sensitivity to material qualities [e.g., threading dislocation (TD)] because of their unique quantum-dot-like localized states from indium-rich regions [10], the power conversion efficiency of InGaN-based $\mathrm{PV}$ devices is still low because of the sensitivity to structural defects in the InGaN absorption layer [2], [6]. To maintain the quality of InGaN alloys utilized in PV devices, the indium

Manuscript received January 5, 2011; accepted January 12, 2011. Date of publication February 24, 2011; date of current version March 23, 2011. This work was supported by the National Science Council under Grant NSC 97-2221-E-006-242-MY3. The review of this letter was arranged by Editor P. K.-L. Yu.

C. C. Yang, J. K. Sheu, M. S. Huang, S. J. Tu, F. W. Huang, X. W. Liang, and W. C. Lai are with the Institute of Electro-Optical Science and Engineering, Advanced Optoelectronic Technology Center, and the Center for Micro/Nano Science and Technology, National Cheng Kung University, Tainan 70101, Taiwan (e-mail: jksheu@mail.ncku.edu.tw).

C. H. Kuo is with the Institute of Lighting and Energy Photonics, National Chiao Tung University, Tainan 71150, Taiwan.

M. L. Lee and Y.-H. Yeh are with the Department of Electro-Optical Engineering, Southern Taiwan University, Tainan 71005, Taiwan (e-mail: minglun@mail.stut.edu.tw).

Color versions of one or more of the figures in this letter are available online at http://ieeexplore.iee.org.

Digital Object Identifier 10.1109/LED.2011.2107725 contents need to be low (e.g., optical band gap around the ultraviolet regions), and the InGaN epilayer thickness needs to be within hundreds of nanometers to avoid generating excess TDs [9]. Several techniques, such as selective area growth and epitaxially lateral overgrowth for GaN/sapphire template substrates, have been developed to reduce the TD density to a lower level $\left(\sim 6 \times 10^{7} \mathrm{~cm}^{-2}\right)$ [11], [12]. However, the epilayer might be as thick as tens of micrometers to achieve a low TD density, thereby smoothing the surface of GaN films. In contrast, backmirror reflectors are utilized to increase the photocurrent up to $18.4 \%$ in the thin absorption layer [2], [4, and references within]. To further increase the photocurrent of InGaN-based PV devices, the indium contents must be raised to a higher level (e.g., visible-light regions) and reach an appropriate absorption layer thickness. In this letter, the sapphire substrates with truncated-cone patterns were used to grow InGaN-based PV devices, and the related PV performance in electrical and optical properties were also shown in this letter.

\section{EXPERIMENTS}

InGaN epitaxial layers were deposited on c-face sapphire substrates by a commercial metal-organic vapor phase epitaxy reactor. Both a conventionally flat sapphire substrate (FSS) and a patterned sapphire substrate (PSS) were simultaneously used during the epitaxy process. The PSSs were prepared via conventional photolithography and dry-etching process. As shown in Fig. 1(a), the PSS featured truncated-cone patterns in the closely triangular package with a spacing of $1.5 \mu \mathrm{m}$. The height, top, and bottom diameters of each pattern were 1.5, 1.0, and $3.0 \mu \mathrm{m}$, respectively. The growth process involved a thin lowtemperature $\mathrm{GaN}$ nucleation layer and a 4.0- $\mu \mathrm{m}$-thick undoped$\mathrm{GaN}$ (u-GaN) layer consisting of a 1.0- $\mu$ m-thick $\mathrm{u}-\mathrm{GaN}$ under a chamber pressure of 500 torr and a 3.0- $\mu$ m-thick $\mathrm{u}-\mathrm{GaN}$ grown under a pressure of 100 torr to feature lateral, coalescence, and quasi-2-D growth models to achieve a smooth surface.

Each step of u-GaN on the PSS is shown in Fig. 1(b) and (c), respectively. After both u-GaN template substrates were prepared, the main $\mathrm{p}$-i-n structure, consisting of a $1-\mu \mathrm{m}$-thick Si-doped $\mathrm{n}^{+}$-GaN layer $\left(\mathrm{n} \sim 5 \times 10^{18} \mathrm{~cm}^{-3}\right)$, an undoped $\mathrm{Al}_{0.14} \mathrm{Ga}_{0.86} \mathrm{~N} / \mathrm{In}_{0.21} \mathrm{Ga}_{0.79} \mathrm{~N}$ (4/3 nm for 14 pairs, respectively) SPS layer, and a $0.1-\mu$ m-thick Mg-doped p-GaN (p $5 \times 10^{17} \mathrm{~cm}^{-3}$ ) layer, was sequentially grown. The alloy contents and the layer thickness were calibrated by $\mathrm{X}$-ray diffraction analyses based on a 100-nm-thick $\mathrm{Al}_{0.14} \mathrm{Ga}_{0.86} \mathrm{~N}$ 

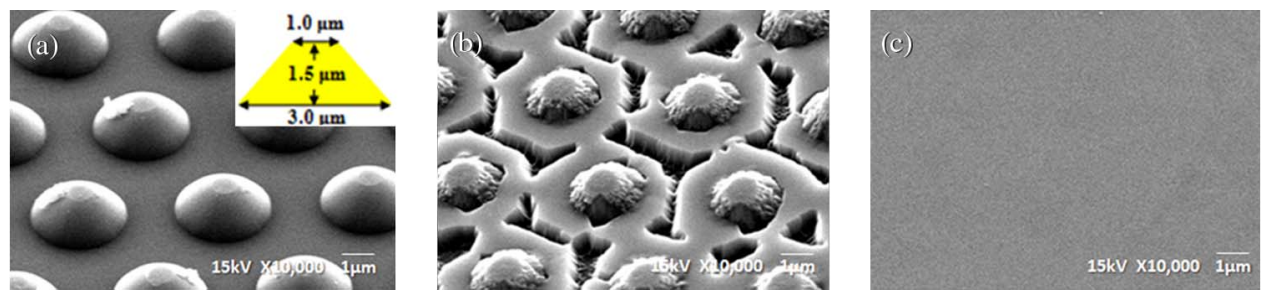

Fig. 1. Typical $45^{\circ}$-tilt scanning-electron-microscope images of (a) the surface morphology of truncated-cone patterns on sapphire substrates, (b) u-GaN under 500 torr, and (c) the coalesced quasi-2-D growth of u-GaN deposited on the PSS.

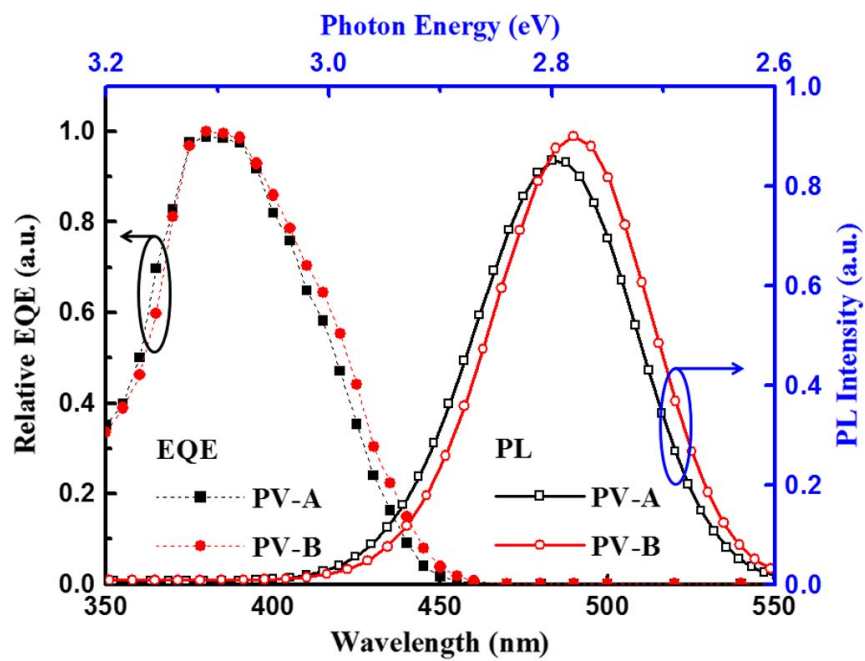

Fig. 2. Relative EQE and room-temperature PL spectra measured by epitaxy structures grown on the FSS (PV-A) and the PSS (PV-B).

bulk layer and a 14-pair $\mathrm{GaN} / \mathrm{In}_{0.21} \mathrm{Ga}_{0.79} \mathrm{~N}$ SPS structure deposited on the u-GaN and/or FSS templates. Samples with epilayer structures deposited on the FSS and the PSS were labeled as PV-A and PV-B, respectively. The device fabrication process was similar to our previous work [6], [13]. Finally, a 200-nmthick $\mathrm{SiO}_{2}$ antireflection layer was coated via plasma-enhanced chemical-vapor deposition. The device area and the electrode coverage rate were $1 \times 1 \mathrm{~mm}^{2}$ and $11.4 \%$, respectively.

\section{Results AND CONCLUSION}

Fig. 2 shows the relative external quantum efficiency (EQE) room-temperature photoluminescence (PL) spectra as a function of incident photon wavelengths and photon energy, respectively. A slight redshift in the absorption edge of PV-B was clearly observed, as compared with PV-A. This is consistent with the PL results, which characterized the peak positions of the PV-A and PV-B samples as 2.793 and $2.780 \mathrm{eV}$ (444 and $446 \mathrm{~nm}$ ), respectively. When the same p-i-n epilayer structure was simultaneously deposited on both substrates, the relatively thinner thickness of $\mathrm{u}-\mathrm{GaN}$ on the PSS resulted in a relatively higher compressive strain (i.e., with an effectively larger c-axial length) [14]. Although this redshift could be beneficial for broader solar-spectrum absorption, it should be carefully considered because the excess strain would eventually relax, generating additional TDs to result in leakage paths in the PV devices.

Fig. 3(a) shows the typical current-density-voltage $(J-V)$ characteristics and solar parameters measured under the global
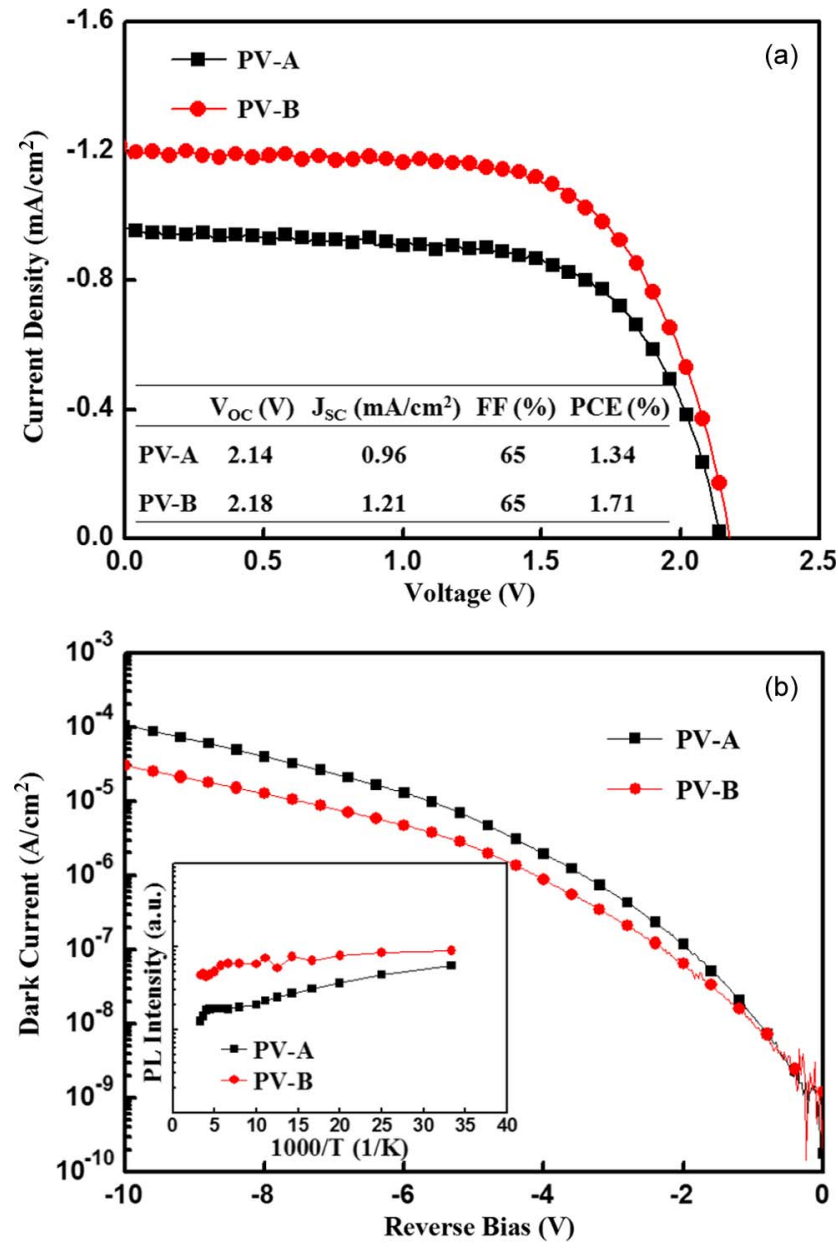

Fig. 3. (a) Typical $J-V$ characteristics of devices taken from the PV cells illuminated under the AM1.5G spectrum. (Inset) Measured PV parameters. (b) The measured dark current density with the reverse bias. (Inset) Arrhenius plots of integrated PL intensities for PV-A and PV-B.

air-mass 1.5 (AM1.5G) irradiance. Noteworthily, the PV-B samples with a relative lower optical band gap exhibited a higher open-circuit voltage $V_{\mathrm{OC}} \cdot V_{\mathrm{OC}}$ is strongly dependent on the saturation current (or the reverse leakage current) of PV devices. At a given reverse bias of $10 \mathrm{~V}$, the dark current density of the PV-B samples was measured to be $3.0 \times 10^{-5} \mathrm{~A} / \mathrm{cm}^{2}$, which is lower than that of PV-A $\left(1.1 \times 10^{-4} \mathrm{~A} / \mathrm{cm}^{2}\right)$, as shown in Fig. 3(b). This improvement could be attributable to the slight reduction of the TD density by the lateral growth on the PSS [15]-[17]. Although a series of concaves on the PSS was filled with the u-GaN epitaxy associated with the bending of TDs, TDs in the epitaxial layers could be the primary factor behind the formation of trap states [18]. The leakage current in the 
GaN/sapphire-based optoelectronic devices mainly originates from defect-related states that produce trap-assisted tunneling and/or recombination. In principle, the PV-B samples with a slightly lower optical band gap, as compared with the PV-A samples, would have a higher short-circuit current density $\left(J_{\mathrm{SC}}\right)$. Compared with the PV-A samples, the calculated enhancement of $J_{\mathrm{SC}}$ is within $10 \%$. However, the measured $J_{\mathrm{SC}}$ of PV-B $\left(1.21 \mathrm{~mA} / \mathrm{cm}^{2}\right)$ was enhanced by $26 \%$, as compared with PV-A $\left(0.96 \mathrm{~mA} / \mathrm{cm}^{2}\right)$. In addition to the small difference in the $E_{g}$ value of InGaN absorption layers between the PV-A and PV-B samples, the increased $J_{\mathrm{SC}}$ of PV-B is attributable to the positive effects of the PSS. To clarify the influence of the PSS on the device efficiency, the relative internal quantum efficiency $\eta_{\text {int }}$ of the PV cells was indirectly evaluated via the intensity integral of temperature-dependent PL spectra [19]. In principle, the thermal quench of luminescence in the absorption layer $\left(\mathrm{Al}_{0.14} \mathrm{Ga}_{0.86} \mathrm{~N} / \mathrm{In}_{0.21} \mathrm{Ga}_{0.79} \mathrm{~N}\right.$ superlattice) is mainly due to the fact that carriers thermally escape from quantized states into the barrier states and/or are captured at nonradiative recombination centers in the active layers. The integrated PL (IPL) intensities as a function of temperature taken from PV-A and PV-B are displayed in the inset of Fig. 3(b). The IPL of both samples gradually declined with the increase in temperature. However, the decline rate of the IPL in PV-A was notably higher than that of PV-B, particularly when the temperatures were close to $300 \mathrm{~K}$. This result indicates that the thermal quench of the IPL resulting from the capture at nonradiative recombination centers in PV-A had a relatively higher probability compared with that of PV-B. This result is consistent with the results that PV-B exhibited relatively higher $J_{\mathrm{SC}}$ and $V_{\mathrm{OC}}$, as shown in Fig. 3(a). The higher $J_{\mathrm{SC}}$ value could be attributed to the fact that photogenerated carriers recombined with defect-related centers in PV-B were relatively less than that in PV-A. On the other hand, the use of the PSS for the GaN/sapphire-based PV cells was beneficial to the reduction of TDs, which, in turn, leads to a reduction of the leakage current and, hence, the increase in $V_{\mathrm{OC}}$ in PV-B.

In conclusion, the InGaN-based PV devices deposited on the PSS with truncated-cone patterns have been demonstrated. Enhancements by approximately $26 \%$ and $2 \%$ in terms of $J_{\mathrm{SC}}$ and $V_{\mathrm{OC}}$, respectively, as compared with the PV devices grown on the FSS, have been obtained. The improved PV parameters could be attributable to the positive effects of the PSS on the material quality.

\section{REFERENCES}

[1] O. Jani, C. Honsberg, A. Asghar, D. Nicol, I. Ferguson, A. Doolittle, and S. Kurtz, "Characterization and analysis of InGaN photovoltaic devices," in Proc. 31st IEEE PVSC, 2005, pp. 37-42.

[2] R. Dahal, J. Li, J. Y. Lin, and H. X. Jiang, "InGaN/GaN multiple quantum well concentrator solar cells," Appl. Phys. Lett., vol. 97, no. 7, p. 073 115, Aug. 2010, and references therein.
[3] O. Jani, I. Ferguson, C. Honsberg, and S. Kurtz, "Design and characterization of GaN/InGaN solar cells," Appl. Phys. Lett., vol. 91, no. 13, p. 132117 , Sep. 2007.

[4] R. H. Horng, M. T. Chu, H. R. Chen, W. Y. Liao, M. H. Wu, K. F. Chen, and D. S. Wuu, "Improved conversion efficiency of textured InGaN solar cells with interdigitated imbedded electrodes," IEEE Electron Device Lett., vol. 31, no. 6, pp. 585-587, Jun. 2010.

[5] J. K. Sheu, C. C. Yang, S. J. Tu, K. H. Chang, M. L. Lee, W. C. Lai, and L. C. Peng, "Demonstration of GaN-based solar cells with GaN/InGaN superlattice absorption layers," IEEE Electron Device Lett., vol. 30, no. 3, pp. 225-227, Mar. 2009.

[6] C. C. Yang, J. K. Sheu, X. W. Liang, M. S. Huang, M. L. Lee, K. H. Chang, S. J. Tu, F. W. Huang, and W. C. Lai, "Enhancement of the conversion efficiency of GaN-based photovoltaic devices with AlGaN/InGaN absorption layers," Appl. Phys. Lett., vol. 97, no. 2, p. 021113 , Jul. 2010

[7] V. Y. Davydov, A. A. Klochikhin, R. P. Seisyan, V. V. Emtsev, S. V. Ivanov, F. Bechstedt, J. Furthmuller, H. Harima, A. V. Mudryi, J. Aderhold, O. Semchinova, and J. Graul, "Absorption and emission of hexagonal InN. Evidence of narrow fundamental band gap," Phys. Stat. Sol. (B), vol. 229, no. 3, pp. R1-R3, Feb. 2002.

[8] J. Wu, W. Walukiewicz, K. M. Yu, J. W. Ager, III, E. E. Haller, H. Lu, W. J. Schaff, Y. Saito, and Y. Nanishi, "Unusual properties of the fundamental band gap of InN,” Appl. Phys. Lett., vol. 80, no. 21, pp. 3967-3969, May 2002.

[9] S. Nakamura, "The roles of structural imperfections in InGaN-based blue light-emitting diodes and laser diodes," Science, vol. 281, no. 5379, pp. 956-961, Aug. 1998.

[10] S. Chichibu, T. Azuhata, T. Sota, and S. Nakamura, "Spontaneous emission of localized excitons in InGaN single and multiquantum well structures," Appl. Phys. Lett., vol. 69, no. 27, pp. 4188-4190, Dec. 1996, and references therein.

[11] A. Sakai, H. Sunakawa, and A. Usui, "Defect structure in selectively grown GaN films with low threading dislocation density," Appl. Phys. Lett., vol. 71, no. 16, pp. 2259-2261, Oct. 1997.

[12] S. Nakamura, M. Senoh, S. Nagahama, N. Iwasa, T. Yamada, T. Matsushita, H. Kiyoku, Y. Sugimoto, T. Kozaki, H. Umemoto, M. Sano, and K. Chocho, "Continuous-wave operation of InGaN/GaN/AlGaNbased laser diodes grown on GaN substrates," Appl. Phys. Lett., vol. 72, no. 16, pp. 2014-2016, Apr. 1998.

[13] M. L. Lee, J. K. Sheu, and C. C. Hu, "Non-alloyed Cr/Au Ohmic contacts to n-GaN," Appl. Phys. Lett., vol. 91, no. 18, p. 182 106, Oct. 2007.

[14] K. Kusakabe, A. Kikuchi, and K. Kishino, "Characterization of overgrown GaN layers on nano-columns grown by RF-molecular beam epitaxy," Jpn. J. Appl. Phys., vol. 40, pt. 2, no. 3A, pp. L192-L194, Mar. 2001, and references therein.

[15] M. Yamada, T. Mitani, Y. Narukawa, S. Shioji, I. Niki, S. Sonobe, K. Deguchi, M. Sano, and T. Mukai, "InGaN-based near-ultraviolet and blue-light-emitting diodes with high external quantum efficiency using a patterned sapphire substrate and a mesh electrode," Jpn. J. Appl. Phys., vol. 41, pt. 2, no. 12B, pp. L1 431-L1 433, Dec. 2002.

[16] S. Watanabe, N. Yamada, M. Nagashima, Y. Ueki, C. Sasaki, Y. Yamada, T. Taguchi, K. Tadatomo, H. Okagawa, and H. Kudo, "Internal quantum efficiency of high-efficient $\operatorname{In}_{x} \mathrm{Ga}_{1-x} \mathrm{~N}$-based near-ultraviolet lightemitting diodes," Appl. Phys. Lett., vol. 83, no. 24, pp. 4906-4908, Dec. 2003.

[17] W. K. Wang, D. S. Wuu, S. H. Lin, P. Han, R. H. Horng, T. C. Hsu, D. T. C. Huo, M. J. Jou, Y. H. Yu, and A. Lin, "Efficiency improvement of near-ultraviolet InGaN LEDs using patterned sapphire substrates," IEEE J. Quantum Electron., vol. 41, no. 11, pp. 1403-1409, Nov. 2005, and references therein.

[18] J. K. Sheu, M. L. Lee, and W. C. Lai, "Effect of low-temperature-grown GaN cap layer on reduced leakage current of GaN Schottky diodes," Appl. Phys. Lett., vol. 86, no. 5, p. 052 103, Jan. 2005, and references therein.

[19] H. W. Huang, J. K. Huang, K. Y. Lee, C. F. Lin, and H. C. Kuo, "Lightoutput-power enhancement of GaN-based light-emitting diodes on an n-GaN layer using a $\mathrm{SiO}_{2}$ photonic quasi-crystal overgrowth," IEEE Electron Device Lett., vol. 31, no. 6, pp. 573-575, Jun. 2010. 